\title{
Nonlinear Conductance-Volume Relationship for Murine Conductance Catheter Measurement System
}

\author{
Chia-Ling Wei*, Member, IEEE, Jonathan W. Valvano, Member, IEEE, Marc D. Feldman, and \\ John A. Pearce, Senior Member, IEEE
}

\begin{abstract}
The conductance catheter system is a tool to determine instantaneous left ventricular volume in vivo by converting measured conductance to volume. The currently adopted conductance-to-volume conversion equation was proposed by Baan, and the accuracy of this equation is limited by the assumption of a linear conductance-volume relationship. The electric field generated by a conductance catheter is nonuniform, which results in a nonlinear relationship between conductance and volume. This paper investigates this nonlinear relationship and proposes a new nonlinear conductance-to-volume conversion equation. The proposed nonlinear equation uses a single empirically determined calibration coefficient, derived from independently measured stroke volume. In vitro experiments and numerical model simulations were performed to verify and validate the proposed equation.
\end{abstract}

Index Terms-Conductance catheter, conductance-volume relationship, volume estimation.

\section{INTRODUCTION}

$\mathbf{P}$ RESSURE-VOLUME analysis is an invasive method for assessing myocardial function. The left ventricular (LV) pressure-volume relationship generated on a beat-by-beat basis during transient occlusion of the inferior vena cava allows hemodynamic characterization of LV systolic and diastolic functions independent of loading conditions [1]-[4]. There is interest in applying LV pressure-volume relationships to characterize the ventricular function in gene-altered mice. However, the measurement of instantaneous volume has been problematic due to the small size of the mouse heart and its rapid rate (up to $700 \mathrm{bpm}$ ). Conductance technology has been miniaturized to generate an instantaneous conductance signal, which is proportional to volume, to solve this problem [5], [6].

Experimentally a four-electrode catheter is inserted into the murine LV to generate an electric field and to continuously

Manuscript received September 26, 2004; revised March 6, 2005. This work was supported in part by the American Heart Association Texas Affiliate under Grant 0255812Y, in part by the National Institutes of Health under Grant HL-67475, and in part by Millar Instruments, Inc., Houston, Texas. Asterisk indicates corresponding author.

*C.-L. Wei was with the Department of Electrical and Computer Engineering, The University of Texas at Austin, Austin, TX 78712 USA. She is now with Silicon Laboratories Inc., 4635 Boston Lane, Austin, TX 78735 USA (e-mail: chia-ling.wei@silabs.com).

J. W. Valvano and J. A. Pearce are with the Department of Electrical and Computer Engineering, The University of Texas at Austin, Austin, TX 78712 USA (e-mail: valvano@mail.utexas.edu; jpearce@mail.utexas.edu).

M. D. Feldman is with the Department of Medicine, The University of Texas Health Science Center at San Antonio, TX 78229 USA (e-mail: feldmanm@uthscsa.edu).

Digital Object Identifier 10.1109/TBME.2005.856029 measure the instantaneous conductance signal. The currently adopted conductance-to-volume conversion equation, proposed by Baan in 1984, is

$$
\begin{aligned}
\mathrm{Vol} & =\frac{1}{\alpha} \rho L^{2}\left(G_{\text {meas }}-G p\right) \\
\Rightarrow \mathrm{Vol} & =\frac{1}{\alpha} \rho L^{2} g_{b}
\end{aligned}
$$

where Vol is the instantaneous volume signal $(l), 1 / \alpha$ is an empirical calibration factor, $\rho$ is the blood resistivity $(\Omega-\mathrm{m})$, $L$ is the distance between the voltage sensing electrodes $(\mathrm{m})$, $G_{\text {meas }}$ is the instantaneous measured conductance (S), $G p$ is the surrounding myocardial conductance $(\mathrm{S})$, and $g_{b}$ is the blood conductance (S) [2]. This conductance-to-volume conversion equation is heretofore referred to as "the classic equation." Experimentally, the stroke volume (SV) is independently measured by an electromagnetic flow probe or Doppler ultrasound, and then $\alpha$ is determined by forcing the volume difference resulting from the conversion equation to be the same as the independently measured SV, i.e.,

$$
\alpha=\frac{\left(\rho L^{2} g_{b-\mathrm{ED}}-\rho L^{2} g_{b-\mathrm{ES}}\right)}{\mathrm{SV}}
$$

where $g_{b-\mathrm{ED}}$ and $g_{b-\mathrm{ES}}$ are blood conductance at end-diastole (ED) and end-systole (ES), respectively. The classic equation is derived from inspection of the volume ( $\mathrm{Vol}$ ) of a cylinder of blood

$$
\mathrm{Vol}=A \cdot L
$$

where $A$ is the cross-sectional area of the cylinder and $L$ is the height. Blood conductance, $\mathrm{g}_{b}$, can be calculated by

$$
g_{b}=\frac{I}{V}=\frac{\oint_{a} \vec{J} \cdot d \vec{a}}{-\int_{l} \vec{E} \cdot d \vec{l}}=\frac{\oint_{a} \sigma \vec{E} \cdot d \vec{a}}{-\int_{l} \vec{E} \cdot d \vec{l}}
$$

where $I$ is current (A), $V$ is voltage (V), $\vec{E}$ is electric field intensity $(\mathrm{V} / \mathrm{m}), \vec{J}$ is current density $\left(\mathrm{A} / \mathrm{m}^{2}\right), a$ is a surface enclosing the source electrode, $l$ is the path length for potential calculation, and $\sigma$ is the blood conductivity (the reciprocal of blood resistivity $\rho$ ) [9]. If the electric field distribution is uniform, which means that the electric field intensity is constant inside the ventricle, (3) can be simplified

$$
g_{b}=\sigma \frac{\vec{E} \cdot A}{\vec{E} \cdot L}=\frac{A}{\rho \cdot L} .
$$

Combining (2) and (4) yields

$$
\mathrm{Vol}=\rho L^{2} g_{b}
$$




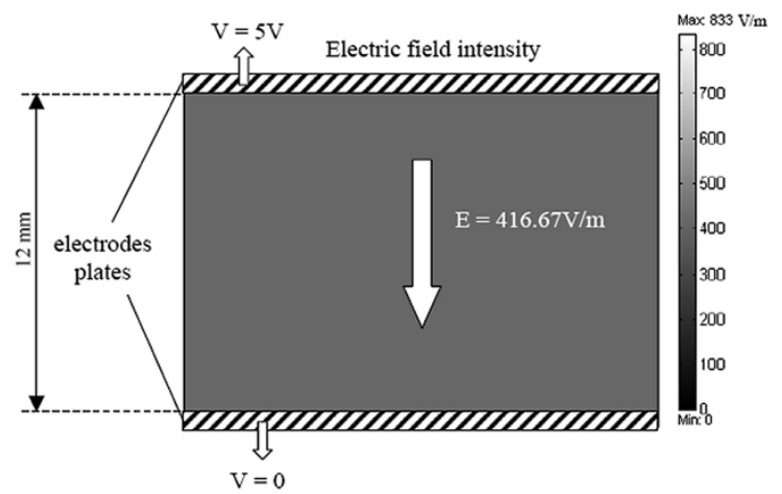

(a)

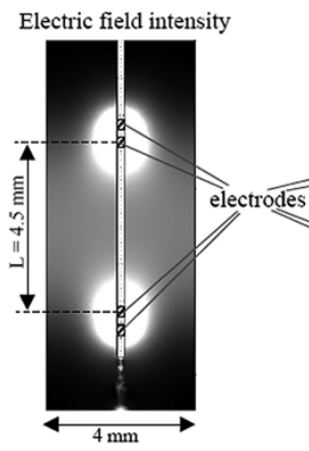

(b)
Electric field intensity

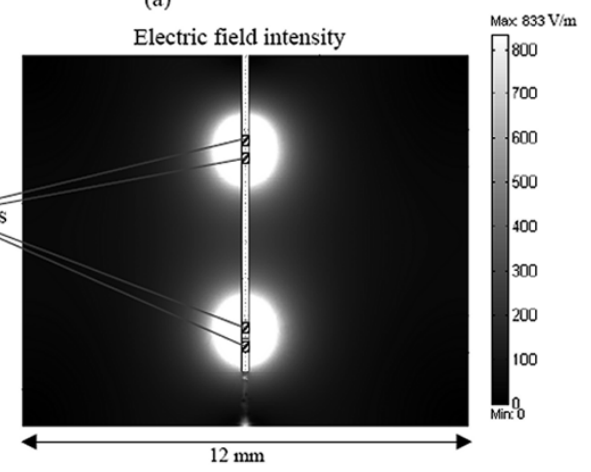

(c)
Fig. 1. The distribution of electric field intensity in a field generated by (a) two large and flat electrode plates (b) a four-electrode catheter placed in a small cylinder-shaped container (c) a four-electrode catheter placed in a large cylinder-shaped container. A voltage difference of $5 \mathrm{~V}$ applies on each source-electrode pairs.

However, a uniform electric field is only achieved when the source electrodes are flat and large enough that fringe effect is negligible [7]-[9]. The electric field generated by LV catheter electrodes is not uniform. Fig. 1 shows the distribution of electric field for both cases, simulated by a commercial finite element package, FEMLAB ${ }^{\circledR}$, Comsol, Inc., MA. Besides, this equation was derived for a cylinder, but the ventricle is not cylindrical. Therefore, in order to compensate for the differences caused by a nonuniform electric field and the noncylinder-shaped ventricle, the empirical factor $1 / \alpha$ is multiplied by (5) to yield (1b), or (1a) if myocardial conductance (Gp) is incorporated.

The accuracy of this ventricular volume estimation method has three limitations [7]. The first problem is the assumption of a linear conductance-volume relationship inherent in the classic equation. In fact, the nonuniform electric field generated by the electrodes makes the conductance-volume relationship nonlinear. This assumption would cause significant errors, especially when estimating larger volumes. The second problem involves the accuracy of estimation of myocardial contribution $(G p)$ to the total measured conductance $\left(G_{\text {meas }}\right)$. The measured conductance $\left(G_{\text {meas }}\right)$ is a combination of the electrical properties of the LV blood and myocardium, since both are conductive. However, only the blood conductance is desired, so the instantaneous parallel myocardium signal should be removed from the total measured conductance. The third problem concerns the positioning of the catheter. During in vivo measurements, deviation of the catheter from the longitudinal axis of the ventricle may introduce errors into the volume measurement.

The second and third problems have been studied extensively and will not be discussed in this paper [2], [7], [10]-[16], but rather the focus will be on the first problem. A new conductance-to-volume conversion equation, which compensates for electric field nonuniformity in a more effective way, is proposed in this paper. Since this paper focuses on the problem of the effect of nonuniform electric field, not including the estimation of myocardial contribution, the proposed equation is a replacement for (1b), which does not include myocardial conductance $(G p)$. Therefore, in vitro experiments with known-volume $\mathrm{KCl}$ solutions placed in cylindrical insulated Plexiglas are appropriate to examine and validate the proposed equation. Furthermore, since the ventricle is not cylindrical, the conductance-volume relation of a cylinder is not exactly the same as the conductance-volume relation in the ventricle. Therefore, it is important to have an empirical factor to calibrate the conductance-volume curve. That is why both the proposed equation and the classic equation have empirical factors. The details of determining the empirical factors are presented in (1c) and Section II.

\section{Methodology}

\section{A. Closed-Form Solution}

The general method for calculating $\vec{E}$ is to solve Laplace's equation, $\nabla^{2} V=0$, in the chosen coordinate system [9], and then obtain $\vec{E}$ by $\vec{E}=-\nabla V$. Once $\vec{E}$ is known, blood conductance $g_{b}$ can be calculated by (3). For a spherical coordinate system $(r, \theta, \varphi)$, the Laplace's equation is

$$
\begin{aligned}
\frac{1}{r^{2}} \frac{\partial}{\partial r}\left(r^{2} \frac{\partial V}{\partial r}\right)+\frac{1}{r^{2} \sin \theta} \frac{\partial}{\partial \theta} & \left(\sin \theta \frac{\partial V}{\partial \theta}\right) \\
& +\frac{1}{r^{2} \sin ^{2} \theta}\left(\frac{\partial^{2} V}{\partial \varphi^{2}}\right)=0
\end{aligned}
$$

Assume that the source electrodes are spheres with radius $a_{0}$ placed in an infinite homogeneous medium. By this simplification, $V$ is independent of $\varphi$, and the standard solution for the axisymmetric form is

$$
V(r, \theta)=\sum_{i=0}^{\infty}\left(A_{i} r^{i}+B_{i} r^{-i-1}\right) P_{i}(\cos \theta)
$$

where $A_{i}$ and $B_{i}$ are coefficients determined by boundary conditions, and $P_{i}(\cos \theta)$ are Legendre polynomials whose values can be obtained from well-established tables. For a single spherical electrode with voltage $V_{0}$ placed in an infinite medium, its boundary conditions can be expressed by

$$
\begin{aligned}
& V\left(a_{0}, \theta\right)=V_{0} \\
& V(\infty, \theta)=0 .
\end{aligned}
$$

Applying the above boundary conditions to (7) yields

$$
\begin{aligned}
& V(r, \theta)=\frac{a_{0} V_{0}}{r} \\
& \vec{E}(r, \theta)=-\nabla V=-\frac{a_{0} V_{0}}{r^{2}} \cdot \vec{r}
\end{aligned}
$$

where $\vec{r}$ represents the radius vector, $r$ is its scalar, and $r \equiv|\vec{r}|$. For two source electrodes with opposite voltage, $V_{0}$ and $-V_{0}$, 


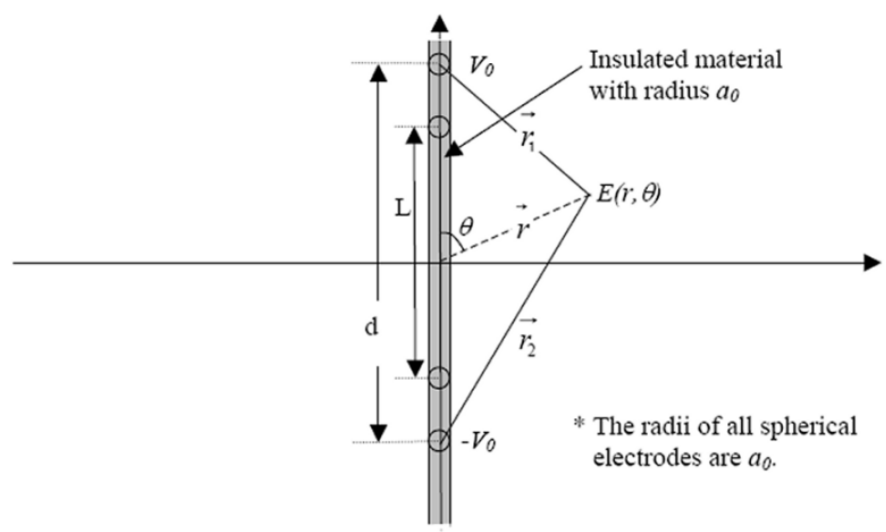

Fig. 2. Two source electrodes and two sensing electrodes are placed in an infinite media.

separated by a distance $d$ (see Fig. 2), the electric field intensity caused by those two electrodes is superposable

$$
\vec{E}(r, \theta)=-\left[\frac{a_{0} V_{0}}{r_{1}^{2}} \cdot \vec{r}_{1}+\frac{a_{0}\left(-V_{0}\right)}{r_{2}^{2}} \cdot \vec{r}_{2}\right]
$$

where

$$
\begin{aligned}
& r_{1} \equiv\left|\vec{r}_{1}\right|=\sqrt{(r \sin \theta)^{2}+\left(\frac{d}{2}-r \cos \theta\right)^{2}} \\
& r_{2} \equiv\left|\vec{r}_{2}\right|=\sqrt{(r \sin \theta)^{2}+\left(\frac{d}{2}+r \cos \theta\right)^{2}} .
\end{aligned}
$$

If two extra voltage-sensing electrodes, which do not carry current, are placed between the two source electrodes separated by a distance $L$ (see Fig. 2), the conductance of this infinite medium ( $\left.g_{\text {inf }}\right)$ measured between the two sensing electrodes can be estimated by using (3) and (11), and the result is

$$
g_{\mathrm{inf}}=\frac{I}{V}=\frac{\frac{2 \pi}{\rho} \int_{a_{0}}^{\infty} \vec{E} \cdot r d \vec{r}}{-\int_{-L / 2}^{L / 2} \vec{E} \cdot d \vec{r}}=\frac{\pi d\left(d^{2}-L^{2}\right)}{4 \rho L \sqrt{\frac{d^{2}}{4}+a_{0}^{2}}} .
$$

It assumes that the radius of the sensing electrodes is small enough so that their influences on the electric field distribution are negligible. In both in vivo and in vitro experiments, the measured fluid is surrounded with either myocardium or a container wall, which confines the distribution of electric field and makes the measured conductance a function of the fluid volume. To further simplify the problem, assume that the measured finite fluid volume is large enough so that the electric field intensity still can be calculated by (11). If the shape of the measured fluid is a cylinder with a radius of $\mathrm{R}$, i.e., its cross-sectional area is $\pi \mathrm{R}^{2}$, then the conductance $g_{b}$ of the measured fluid is

$$
\begin{aligned}
g_{b} & =\frac{I}{V}=\frac{\frac{2 \pi}{\rho} \int_{a_{0}}^{R} \vec{E} \cdot r d \vec{r}}{-\int_{-L / 2}^{L / 2} \vec{E} \cdot d \vec{r}} \\
& =\frac{\pi d\left(d^{2}-L^{2}\right)}{4 \rho L} \cdot\left(\frac{1}{\sqrt{a_{0}^{2}+\left(d^{2} / 4\right)}}-\frac{1}{\sqrt{R^{2}+\left(d^{2} / 4\right)}}\right) .
\end{aligned}
$$

Combining (2), (13), and (14) yields

$$
\mathrm{Vol}=\frac{\pi L d^{2} g_{\mathrm{inf}}^{2}}{4}\left[\frac{1+4 a_{0}^{2} / d^{2}}{\left(g_{\mathrm{inf}}-g_{b}\right)^{2}}-\frac{1}{g_{\mathrm{inf}}^{2}}\right] \text {. }
$$

Equation (15) is derived from a cylinder, but the shape of ventricle is not cylindrical. This equation does not have an empirical factor, like $1 / \alpha$ in the classic equation, to force volume differences resulting from the smallest and largest blood conductance ( $g_{b-\mathrm{ES}}$ and $\left.g_{b-\mathrm{ED}}\right)$ the same as independently measured $\mathrm{SV}$. Therefore, an empirical factor, $\beta$, must be introduced to approach this drawback wherein all of the dimensional constants $(L, d$, etc.) are included in this factor. Furthermore, since the radius of the electrodes $\left(a_{0}\right)$ is small compared to the distance between the two source electrodes $(d)$, the factor $\left(1+4 a_{0}^{2} / d^{2}\right)$ in (15) is approximately 1 . The improved equation, which is heretofore referred to as "the analytic approximation," is

$$
\mathrm{Vol}=\frac{\beta}{\left(g_{\text {inf }}-g_{b}\right)^{2}}-\frac{\beta}{g_{\text {inf }}^{2}}
$$

where the value of $\beta$ can be determined experimentally by

$$
\beta=\mathrm{SV} \cdot\left[\frac{1}{\left(g_{\mathrm{inf}}-g_{b-\mathrm{ED}}\right)^{2}}-\frac{1}{\left(g_{\mathrm{inf}}-g_{b-\mathrm{ES}}\right)^{2}}\right]^{-1} \text {. }
$$

This analytic approximation was derived under several unrealistic assumptions. However, without these assumptions to simplify the problem, a true analytic solution may not be calculable. Hence, an empirical method was used to derive the conductance-volume relationship in the following sections.

\section{B. In Vitro KCl Solution Experiments and Simulations}

In vitro experiments were performed in $\mathrm{KCl}$ solution to examine the conductance-volume relationship of the conductance catheter. Several cylindrical holes were drilled in thick blocks of Plexiglas. The conductivity of the Plexiglas is essentially zero. The conductivity of $\mathrm{KCl}$ solution $(0.1$ mole of $\mathrm{KCl}$ in 1 liter of water) used to fill those holes was $1.26 \mathrm{~S} / \mathrm{m}$ at 23 ${ }^{\circ} \mathrm{C}$. Both miniaturized mouse (SPR-839) and rat (SPR-838) conductance catheters made by Millar Instruments, Houston, Texas, were used to evaluate the conductance-volume relationship in different volume ranges. The mouse catheter has four $0.25-\mathrm{mm}$-long platinum ring electrodes with $0.2-\mathrm{mm}$ radius. The interelectrode center-to-center spacing for the mouse catheter is $0.5,4.5$, and $0.5 \mathrm{~mm}$, respectively. The rat catheter has four $0.25-\mathrm{mm}$-long platinum ring electrodes with $0.2-\mathrm{mm}$ radius. The interelectrode spacing for the rat catheter is 0.5 , 9.0 , and $0.5 \mathrm{~mm}$, respectively. The true volume is defined as the solution volume between the two inner voltage-sensing electrodes.

The catheter was inserted into the center of the cylindrical hole and fixed using a mechanical holder. It is important for reproducibility for the catheter to be exactly in the center. The block of plexiglas was placed on a platform whose height is adjustable to control the depth of catheter insertion. The catheter depth is defined as the distance between the fluid surface and the top of the top electrode. A constant $10-\mathrm{kHz}, 30-\mu \mathrm{A}$ peak excitation ac current was applied to the two outermost electrodes to generate the electric field. The voltage difference between two inner electrodes was measured. The magnitude of the voltage signal from the two inner electrodes is proportional 


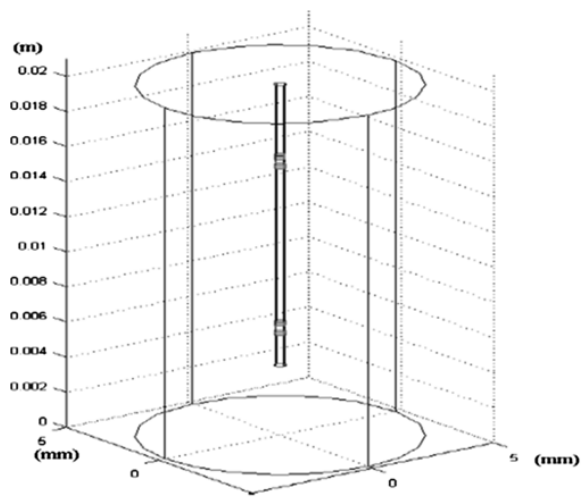

(a)

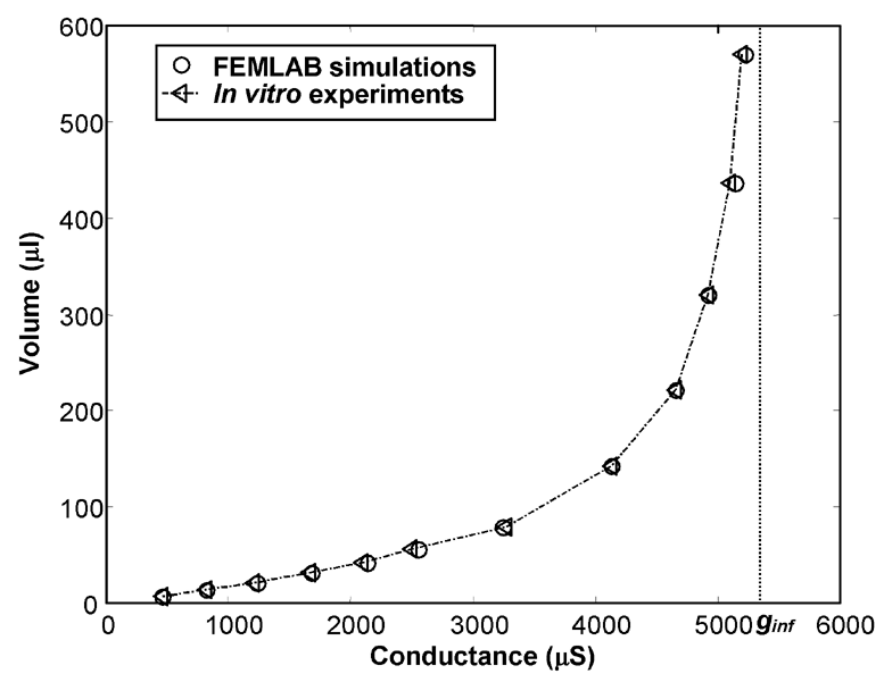

(c)

Fig. 3. (a) A 3-D FEMLAB model (b) cross-sectional view (c) the conductance-volume plot of FEMLAB simulation and in vitro $\mathrm{KCl}$ experimental data using the rat catheter.

to the resistance between the inner electrode pair. This signal is then amplified and rectified to remove the carrier frequency, $10 \mathrm{kHz}$. The reciprocal of this signal was taken to yield the conductance-magnitude-proportional voltage output, calibrated to conductance.

Numerical finite element models were constructed using FEMLAB $^{\circledR}$ to mimic the in vitro $\mathrm{KCl}$ solution experiments. The solution-filled cylindrical holes are modeled with cylinders in FEMLAB, which have insulated boundaries and corresponding radiuses. The center of the cylinders along the $\mathrm{z}$ axis is emptied in order to model the insulated catheter tube. Four small cylinders with conductivity of $9661000 \mathrm{~S} / \mathrm{m}$ (platinum) are used to model the metal electrodes [Fig. 3(a), (b)]. The inner pair is to model the floating voltage-sensing electrodes, so all their boundaries are insulated. The outer pair is to model the current source electrodes: one is grounded, and the other one has a current density of $238.7 \mathrm{~A} / \mathrm{m}^{2}$ on one 2-mm-radius circular boundary, which gives $30 \mu \mathrm{A}$ totally to the KCL solution. The finite elements used in this paper are quadratic, and a linear iterative solver with drop tolerance of $10^{-5}$ was used.

The measured solution conductance corresponds to blood conductance $\left(g_{b}\right)$ in in vivo experiments, so we still use $g_{b}$ to represent the measured solution conductance in the following

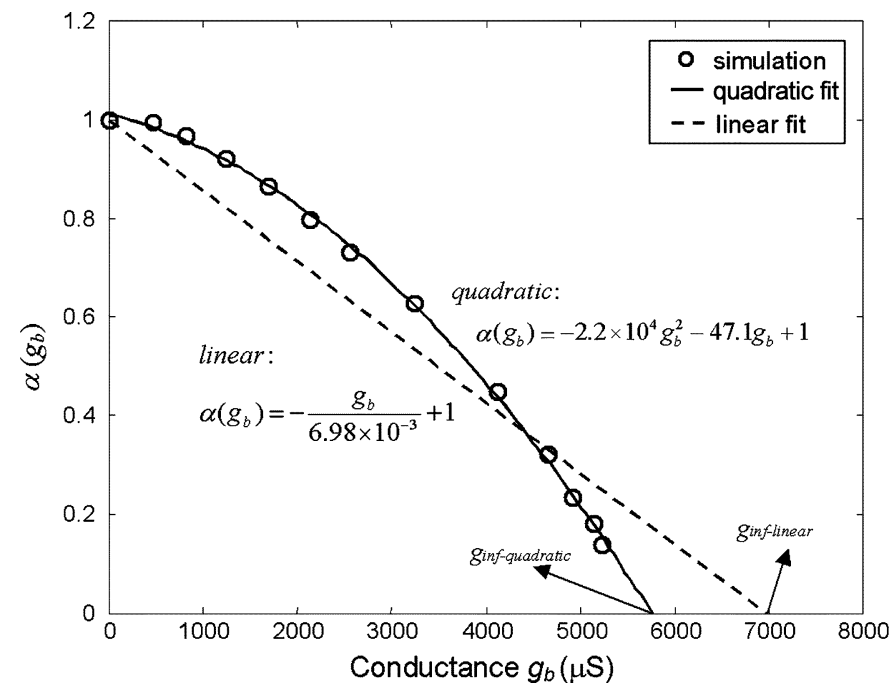

Fig. 4. The conductance $g_{b}$ versus $\alpha\left(g_{b}\right)$ plot of FEMLAB simulation data.

sections. The equivalent SV in the plexiglas experiments and simulations is the difference between the largest and smallest measured solution volumes. Both the experimental and simulation results show that the conductance-volume relationship is curved, not linear [Fig. 3(c)]. Each point of the experimental data in Fig. 3(c) is the average of three measurements using the mouse catheter.

\section{Empirical and Numerical Analysis}

Experimentally, when the measured volume increases enormously, the catheter-measured conductance approaches a saturation value, denoted as ginf [Fig. 3(c)]. Theoretically, if the radius of the container approaches zero, the catheter is surrounded with a nonconductive Plexiglas wall. In that instance, neither current nor electric fields exist between the electrodes, which results in zero measured conductance. Furthermore, the electric field distribution between the two inner sensing electrodes is more uniform when the radius of the container is very small [Fig. 1(b), (c)]. Therefore, as the cylinder shrinks to approach the catheter size, the conductance-to-volume relationship should approach the equation for a uniform electric field distribution, i.e., (5). From the above observations, the conductance-volume relationship of the four-electrode catheter must satisfy the following three conditions:

1) has an asymptote $g_{b}=g_{\text {inf }}$ as volume approaches infinity;

2) goes through the origin, i.e., Vol is zero when $g_{b}$ is zero;

3) approaches (5) at small volume range, i.e.,

$$
\left.\frac{d \mathrm{Vol}}{d g_{b}}\right|_{g_{b}=0}=\rho L^{2} .
$$

There are many possible equations that meet these three criteria. We tried to find a simple one that not only satisfies those criteria but also can accurately predict the conductancevolume relationship. In the classic equation, $\alpha$ is fixed, so the conductance-volume relationship is linear. If $\alpha$ is a function of measured conductance $\left(g_{b}\right)$, not a constant, the equation has the potential to be nonlinear. Fig. 4 shows the $g_{b}-\alpha$ plot of FEMLAB $^{\circledR}$ simulation results. 


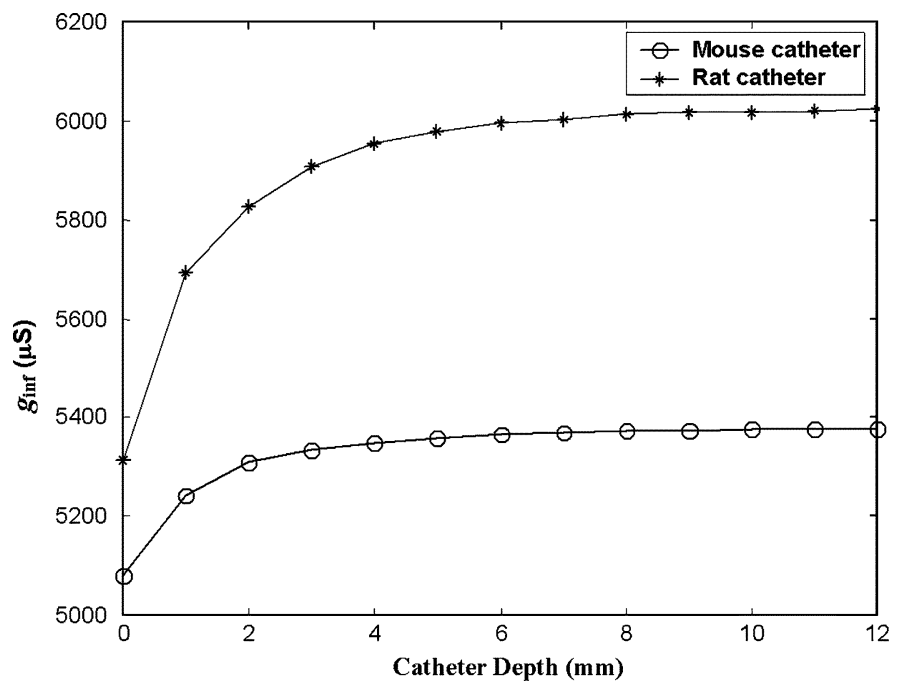

Fig. 5. Plot of maximum measurable conductance $g_{\text {inf }}$ versus catheter depth for the mouse and rat catheters.

The simulation data can be well-fit by a quadratic polynomial, which has at least two coefficients to be determined. However, in in vivo measurements, only the $\mathrm{SV}$, measured by an electromagnetic flow probe, is available to calibrate the equation, which means that only one undetermined coefficient is allowed. Therefore, a linear approximation is used. The linear regression line shown in Fig. 4 fits the simulation data reasonably well in this mouse- and rat-sized LV volume range, although $g_{\text {inf }}$ estimated from the quadratic and linear equations are different. Hence, a new equation is proposed

$$
\mathrm{Vol}=\frac{1}{\alpha\left(g_{b}\right)} \rho L^{2} g_{b}=\frac{g_{\mathrm{inf}}}{g_{\mathrm{inf}}-g_{b}} \rho L^{2} g_{b}
$$

where

$$
\alpha\left(g_{b}\right)=1-\frac{g_{b}}{g_{\text {inf }}}
$$

When $g_{b}$ approaches $g_{\text {inf }}, \alpha\left(g_{b}\right)$ approaches zero, which makes Vol in (17a) increase without bound, meeting the first criterion. When $g_{b}$ is zero, Vol is forced to zero, which satisfies the second criterion. Further, when $g_{b}$ approaches zero, the derivative of (17a) with respect to $g_{b}$ is $\rho L^{2}$, meeting the third criterion.

However, (17a) has a severe drawback: it does not have an empirical factor to calibrate the independently measured SV. As it is stated above, an empirical factor is needed in order to accurately estimate the volume of a noncylindrical object.

On the other hand, the parameter, $g_{\text {inf }}$, in (17a) is very difficult to determine. Experimentally, $g_{\text {inf }}$ is a function of catheter depth for shallow insertions of the catheter (see Fig. 5). It is because the upper electric field is confined and then affects the measured conductance. The electric field intensity decreases dramatically with increasing distance, so the effects coming from electric fields far away from the catheter are negligible. Therefore, once the submerged depth of the catheter exceeds a critical level, the measured conductance is independent of catheter depth. In in vivo murine experiments, it is impossible to keep the catheter away from the aortic valve, which means that the electric field will be confined or affected. As a result, the $g_{\text {inf }}$ estimated from

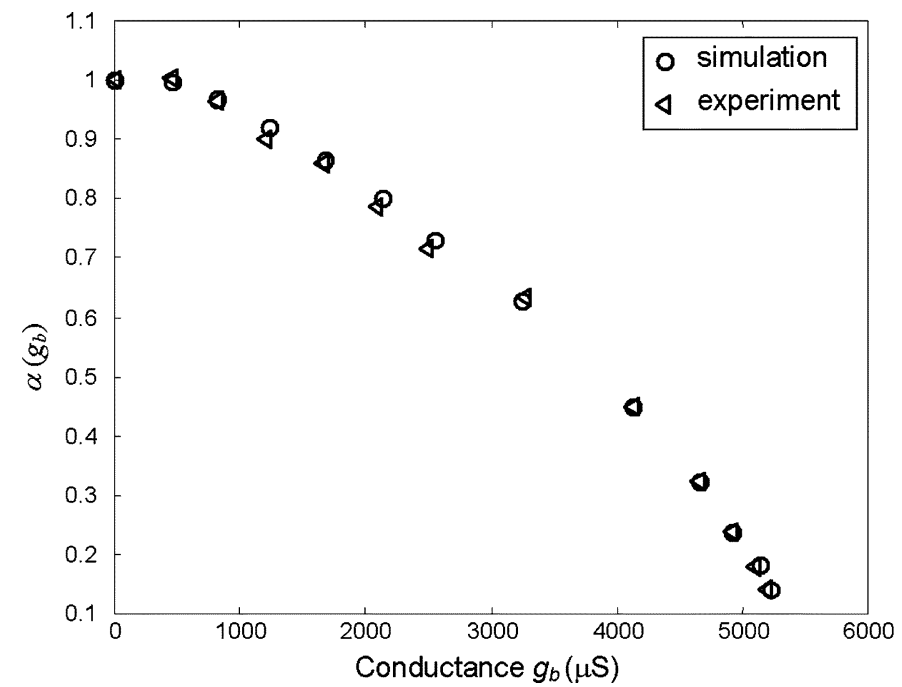

Fig. 6. Comparison of finite element simulation and in vitro $\mathrm{KCl}$ experimental data in conductance $g_{b}$ versus $\alpha\left(g_{b}\right)$ plot, where each experimental point is the average of three measurements.

in vitro saline experiments may be different from the real $g_{\text {inf }}$ in vivo. This small error causes a large effect in the results.

To solve these two problems, $g_{\text {inf }}$ is replaced by an empirical factor, $\gamma$, and (17a) then becomes

$$
\mathrm{Vol}=\frac{1}{\alpha\left(g_{b}\right)} \rho L^{2} g_{b}=\frac{\gamma}{\gamma-g_{b}} \rho L^{2} g_{b}
$$

where

$$
\alpha\left(g_{b}\right)=1-\frac{g_{b}}{\gamma}
$$

Equation (18a) is heretofore referred to as "the empirical approximation." The empirical factor, $\gamma$, is determined by forcing the calculated SV from the empirical approximation to be the same as the independently measured SV. Mathematically, it can be calculated by

$$
\gamma=\frac{-b \pm \sqrt{b^{2}-4 a c}}{2 a}
$$

where

$$
\begin{aligned}
& a=\mathrm{SV}-\rho L^{2}\left(g_{b-\mathrm{ED}}-g_{b-\mathrm{ES}}\right) \\
& b=-\mathrm{SV} \cdot\left(g_{b-\mathrm{ED}}+g_{b-\mathrm{ES}}\right) \\
& c=\mathrm{SV} \cdot g_{b-\mathrm{ED}} \cdot g_{b-\mathrm{ES}} .
\end{aligned}
$$

The larger positive solution for $\gamma$ is appropriate.

\section{RESULTS}

Fig. 6 compares the finite element simulation and in vitro $\mathrm{KCl}$ experimental data in a $g_{b}-\alpha$ plot, where each experimental point is the average of three measurements. The experimental data are consistent with the simulation results.

Fig. 7(a), (b) shows $g_{b}$-Vol plots comparing the experimental data, measured by the mouse and rat catheters, respectively, with the classic, analytic, and empirical approximation equations. The volume ranges of these two experiments correspond to the LV volume seen in mice and rats, respectively. The catheter depths are 6 and $4 \mathrm{~mm}$ for the mouse and rat catheters, respectively. Both figures show that the classic equation overestimates 


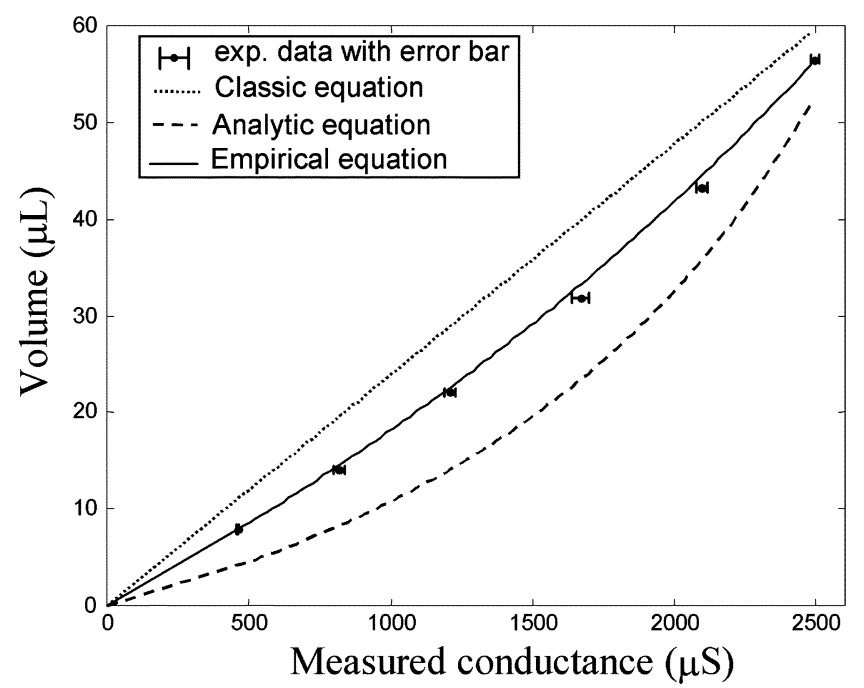

(a)

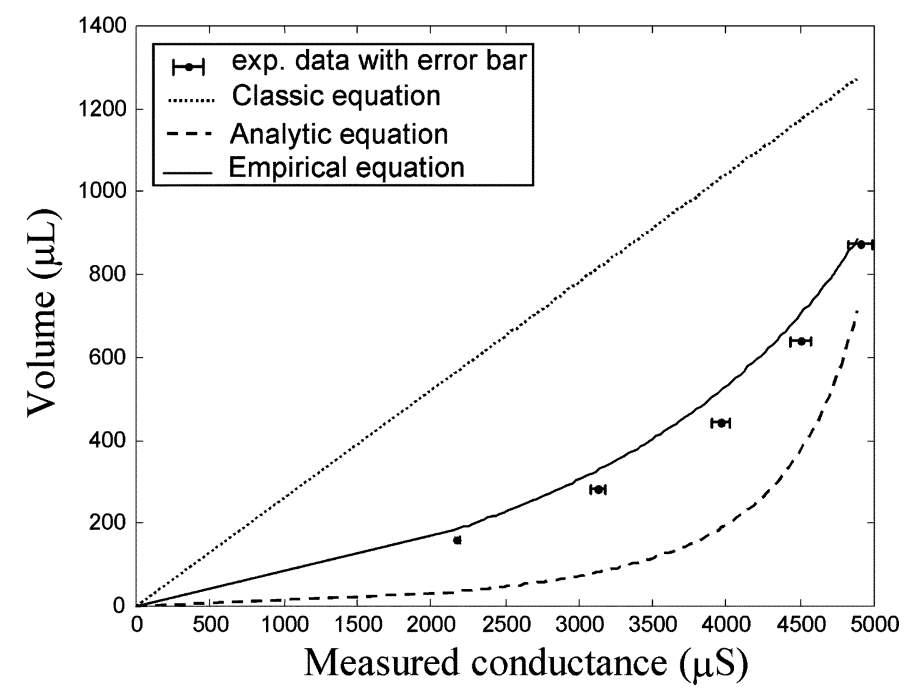

(b)

Fig. 7. Comparison of measured data with the classic, analytic, and empirical approximation equations in a conductance-volume plot. These data were measured by (a) the mouse catheter and (b) the rat catheter.

the volumes and the analytic approximation underestimates the volumes, while the empirical approximation is closest to the true volumes. The classic equation overestimates volume by an average of $492 \mu \mathrm{l}$ in the rat catheter experiment and $5.5 \mu \mathrm{l}$ in the mouse catheter experiment, while the volume error estimated by the empirical equation is only $46 \mu \mathrm{l}$ in the rat catheter experiment and $0.6 \mu \mathrm{l}$ in the mouse experiment. The volume error is reduced to only about $10 \%$ of the original value by use of the empirical equation.

\section{DISCUSSION}

The nonuniform electric field generated by the catheter electrodes makes the relationship between measured conductance $\left(g_{b}\right)$ and its corresponding volume (Vol) nonlinear. The classic conductance-to-volume conversion equation is linear. So, one reason to introduce the empirical factor, $\alpha$, is trying to fit the inherently nonlinear conductance-volume relationship

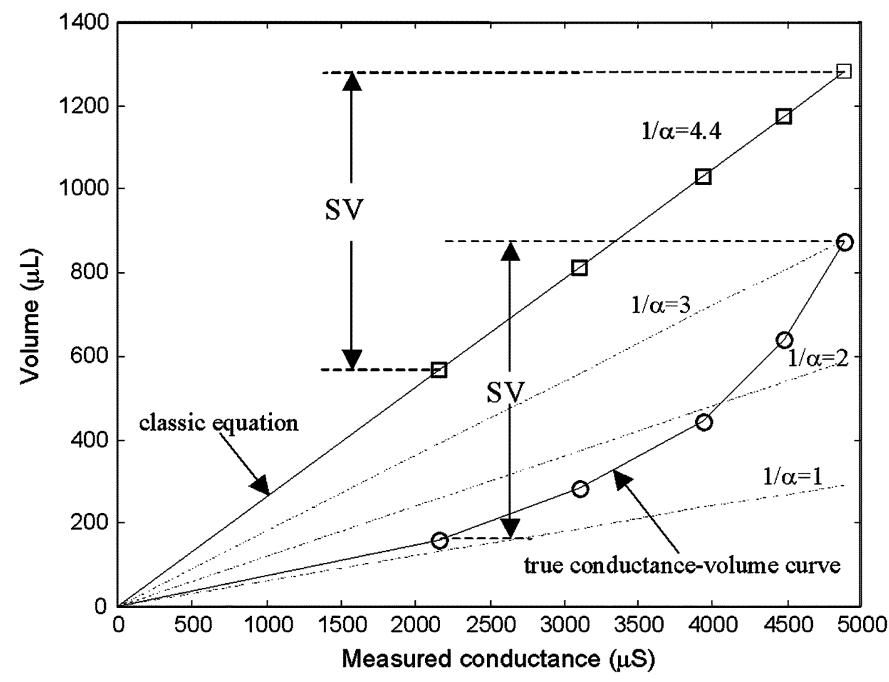

Fig. 8. Plot of true conductance-volume curve versus the classic equations with different values of $\alpha$. The volume range is seen in rat LV and the data were collected using the rat catheter.

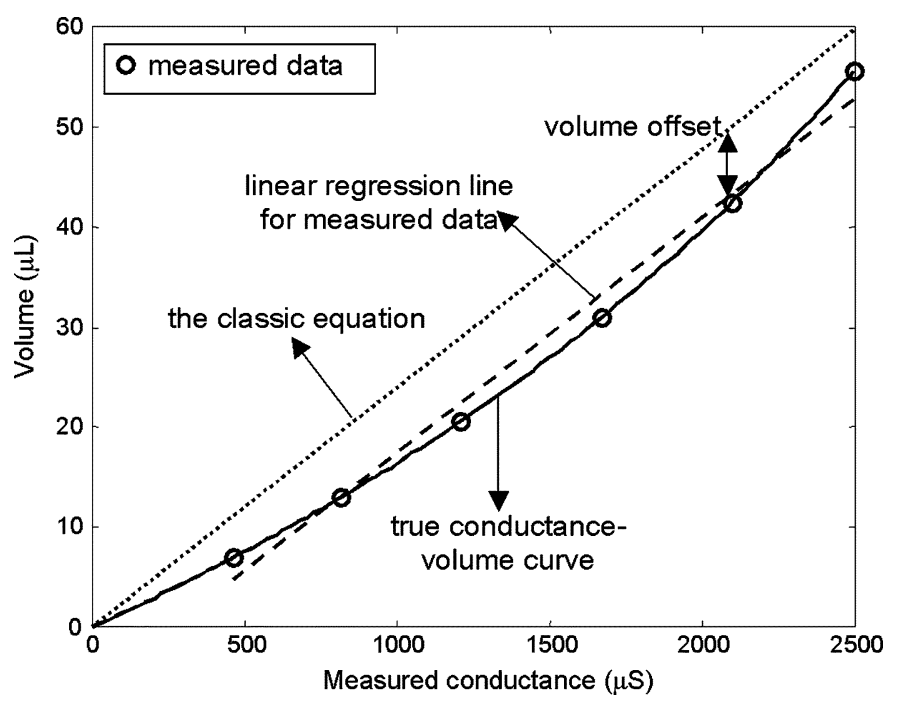

Fig. 9. Comparison of the classic equation, the true conductance-volume curve, the measured data (circles) and its regression line. The volume range is seen in mouse LV and the data were collected using the mouse catheter.

by changing the conductance-volume slope to match the independently measured SV [2]. As a matter of fact, $(1 / \alpha)=1$ is for the uniform electric field, but the slope of the true conductance-volume curve for this nonuniform electric field is not constant. Actually, the slope gets larger as conductance increases [see Fig. 3(c)]. Therefore, $(1 / \alpha)$ is always larger than 1 and the classic conversion equation tends to overestimate volume, as is illustrated in Fig. 8, which uses the data from the rat-sized volume experiment. The value of $1 / \alpha, 4.4$, is determined using (1c). This volume-overestimated situation gets worse in the larger conductance-volume range, in which true volume increases rapidly as conductance only increases slightly [Fig. 3(c)]. The experiments show that the accuracy of the classic conversion equation has much room to be improved in the range of volumes seen in a rat LV [see Fig. 7(b)]. In the range of volumes seen in a mouse $\mathrm{LV}$, the estimation of the classic equation is acceptable with a small volume offset (see Fig. 9). Although the relationship between conductance and 
volume is approximately linear in the mouse-sized LV range, the slope of its regression line is still larger than 1 , which means $1 / \alpha$ is also larger than 1 . However, the classic equation forces volume to be zero when measured conductance is zero (the classic equation: $\mathrm{Vol}=(1 / \alpha) \rho L^{2} g_{b}$, if $\left.g_{b}=0, \mathrm{Vol}=0\right)$. Therefore, it still overestimates volume by a volume offset in the mouse-sized LV range, which is illustrated in Fig. 9.

The analytic approximation (16a), (16b) and the empirical approximation (18a)-(18c) are both nonlinear. Their slopes in conductance-volume plot vary with measured conductance, and are not a fixed number. This feature makes them more attractive in larger volume range, such as LV seen in rats or mice with LV hypertrophy. However, the performance of the analytic approximation is not satisfying, since the analytic approximation was derived under the assumption that the electrodes are placed in a large medium. In both in vivo and in vitro experiments, the catheter is actually placed in a finite and very limited space. In addition, the boundary conditions introduced by the finite volume of measured fluid would result in many nonzero high-order terms in the analytic solution of Laplace's equation, (7), which means that (11) is too simplistic to describe the real electric field distribution. Therefore, the relationship between conductance and volume is actually much more complicated than the analytic approximation. Since a true analytic solution might not be calculable, the empirical analysis was performed.

From Fig. 7, the real conductance-volume relationship falls between the analytic approximation and the classic equation, and the curve of empirical equation is between the two equations and well predicts the true conductance-volume relationship in both mouse and rat LV volume ranges.

To apply the proposed equation on in vivo murine experiments, we propose an experimental procedure: one can measure and record the conductance signals in the LV first, and then use an aortic flow probe to measure SV independently. After these measurements are done, one bleeds the mouse and places the blood in a test tube in a water bath at $37^{\circ} \mathrm{C}$. One then measures its conductance with the conductance catheter centered in the test tube, and converts it to blood resistivity by use of a conductance-to-resistivity lookup table built from in vitro saline experiments. Another alternative method is to use the mean value of blood resisitivities measured from a subset of mice in the same strain. Once the parallel myocardial conductance is estimated using techniques published in the previous literature [2], [10]-[12], [16], blood conductance can be obtained by subtracting the estimated myocardial conductance from the total measured conductance. Then, one determines the empirical factor $\gamma$ from (18c), so that the resulting SV from the empirical approximation equation will be the same as the SV measured by the aortic flow probe. Finally, the blood conductance is converted to the $L V$ volume by use of the empirical approximation equation, (18a). If myocardial conductance is incorporated, the empirical approximation equation becomes

$$
\begin{aligned}
\mathrm{Vol} & =\frac{\gamma}{\gamma-g_{b}} \rho L^{2} g_{b} \\
& =\frac{\gamma}{\gamma-\left(G_{\text {meas }}-G p\right)} \rho L^{2}\left(G_{\text {meas }}-G p\right) .
\end{aligned}
$$

Because of the need to measure the resistivity of the mouse blood, the actual conductance-to-volume conversion is done off-line.

\section{CONCLUSION}

The effects of the nonuniform electric field on the conductance-volume relationship was explored and discussed. The nonlinear conductance-volume relationship caused by the nonuniform electric field has been analyzed using both fundamental electromagnetic theory and an empirical method. Since the classic equation is inherently linear, it cannot predict the conductance-volume relationship very well, especially in larger volume range, e.g., LV seen in rats. Even in the mouse LV volume range, a volume offset is unavoidable by use of the classic equation. If the empirical equation is used instead, volume error can be reduced to only about $10 \%$ of the original value based on the data presented in this paper. In conclusion, the nonlinear empirical conductance-to-volume equation showed the best overall performance and is recommended for use.

\section{REFERENCES}

[1] D. Kass, M. Midei, W. Graves, J. Brinker, and W. L. Maughan, "Use of a conductance (volume) catheter and transient inferior vena caval occlusion for rapid determination of pressure-volume relationships in man," Cath. Card. Diag., vol. 15, pp. 192-202, 1988.

[2] J. Baan et al., "Continuous measurement of left ventricular volume in animals and humans by conductance catheter," Circ., vol. 70, pp. 812-823, 1984.

[3] K. Sagawa, L. Maughan, H. Suga, and K. Sunagawa, Cardiac Contraction and the Pressure-Volume Relationship. New York: Oxford Univ. Press, 1988.

[4] H. Suga, "Cardiac energetics: From Emax to pressure volume area (review)," Clin. Exp. Pharm. Physiol., vol. 30, pp. 580-585, 2003.

[5] D. Georgakopoulos, W. A. Mitzner, C. H. Chen, B. J. Byrne, H. D. Millar, J. M. Hare, and D. A. Kass, "In vivo murine left ventricular pressure-volume relations by miniaturized conductance micromanometry," Am. J. Physiol. Heart Circ. Physiol., vol. 274, pp. H1416-H1422, 1998.

[6] M. D. Feldman, J. M. Erikson, Y. Mao, C. E. Korcarz, R. M. Lang, and G. L. Freeman, "Validation of a mouse conductance system to determine LV volume: Comparison to echocardiography and crystals," Am. J. Physiol. Heart Circ. Physiol., vol. 279, pp. H1698-H1707, 2000.

[7] C. C. Wu, T. C. Skalak, T. R. Schwenk, C. M. Mahler, A. Anne, P. W. Finnerty, H. L. Haber, R. M. Weikle, and M. D. Feldman, "Accuracy of the conductance catheter for measurement of ventricular volumes seen clinically: Effects of electric field homogeneity and parallel conductance," IEEE Trans. Biomed. Eng., vol. 44, no. 4, pp. 266-277, Apr. 1997.

[8] C. L. Wei, J. W. Valvano, M. D. Feldman, M. Nahrendorf, and J. A. Pearce, "3D finite element complex domain numerical models of electric fields in blood and myocardium," in Proc. IEEE-Engineering in Medicine and Biology. Soc., 25th Annu. Int. Conf., Sep. 2003, pp. 62-65.

[9] D. K. Cheng, Field and Wave Electromagnetics. Reading, MA: Addison-Wesley, 1989, pp. 198-219.

[10] M. D. Feldman, Y. Mao, J. W. Valvano, J. A. Pearce, and G. L. Freeman, "Development of a multifrequency conductance catheter-based system to determine LV function in mice," Am. J. Physiol. Heart Circ. Physiol., vol. 279, pp. H1411-H1420, 2000.

[11] D. Georgakopoulos and D. A. Kass, "Estimation of parallel conductance by dual-frequency conductance catheter in mice," Am. J. Physiol. Heart Circ. Physiol., vol. 279, pp. H443-H450, 2000.

[12] T. S. Gawne, K. S. Gray, and R. E. Goldstein, "Estimating left ventricular offset volume using dual frequency conductance catheters," J. Appl. Physiol., vol. 63, no. 2, pp. 872-876, 1987.

[13] P. A. White et al., "The effect of changing excitation frequency on parallel conductance in different sized hearts," Cardiovasc. Res., vol. 38, pp. 668-675, 1998.

[14] J. C. Woodard, C. D. Bertram, and B. S. Gow, "Effect of radial position on volume measurements using the conductance catheter," Med. Biol. Eng. Comput., vol. 27, pp. 25-32, 1989.

[15] S. Kun and R. A. Peura, "Analysis of conductance volumetric measurement error sources," Med. Biol. Eng. Comput., vol. 32, pp. 94-100, 1994. 
[16] E. B. Lankford, D. A. Kass, W. L. Maughan, and A. A. Shoukas, "Does volume catheter parallel conductance vary during a cardiac cycle?," Am. J. Physiol. Heart Circ. Physiol., vol. 258, pp. H1933-H1942, 1990.

[17] J. A. Pearce and M. D. Feldman, "Numerical model study of dual frequency conductance measurement of left ventricular volume in the mouse," presented at the IEEE-Engineering in Medicine and Biology Soc., 24th Annu. Meeting, Oct. 2002, Poster.

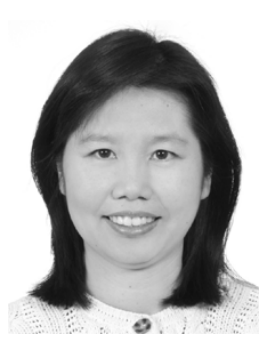

Chia-Ling Wei (S'01-M'04) received the B.S. and M.S. degrees both in electrical engineering from National Taiwan University, Taipei, Taiwan, in 1995 and 1997, and the Ph.D. degree in electrical and computer engineering from The University of Texas at Austin, Austin, TX, in 2004. Her dissertation focused on the estimation of murine cardiac volume by use of a conductance catheter.

She was with Taiwan Semiconductor Manufacturing Company Ltd., Hsinchu, Taiwan, from 1997 to 1999 , where she worked on integrated circuit design. In 2004, she joined Silicon Laboratories Inc., Austin, TX, where she is currently a Design Engineer.

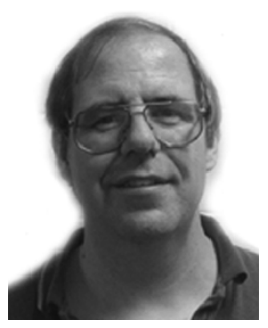

Jonathan W. Valvano (M'83) was born in Clinton, CT, in 1953. He received the B.S. degree in computer science and engineering and the M.S. degree in electrical engineering and computer science from the Massachusetts Institute of Technology, Cambridge, in 1977. He received the Ph.D. degree in medical engineering from the Harvard University/MIT Division of Health Sciences and Technology in 1981.

$\mathrm{He}$ is currently a Full Professor at The University of Texas at Austin, performing research in the fields of perfusion measurements, bioinstrumentation, and

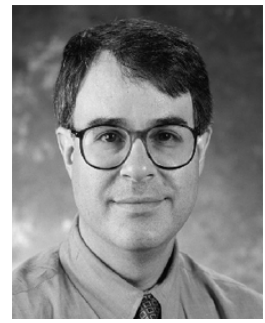

Marc D. Feldman received the B.S. degree from the Duke University, Durham, NC, in 1977, and the M.D. degree from the University of Pennsylvania School of Medicine, Philadelphia, in 1981.

He completed a Medical Residency at the University of Chicago, IL. He took a Fellowship in Cardiology at Beth Israel Hospital, Harvard Medical School, Cambridge, MA and joined the Faculty of the University of Virginia, Charlottesville, where he became an Associate Professor of Medicine and Director of Inpatient Cardiology. He was also an Associate Professor of Medicine and Director of the Cardiac Catheterization Laboratory at the University of Pittsburgh, Pittsburgh, PA. He is currently Associate Professor of medicine and Physiology at the University of Texas Health Science Center at San Antonio. His interests include development of instrumentation as it relates to cardiovascular disease.

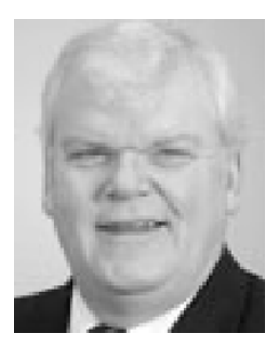

John A. Pearce (M'78-SM'89) received the B.S.M.E. and M.S.M.E. degrees from Clemson University, Clemson, SC, in 1968 and 1971, respectively, and the M.S.E.E. and Ph.D. degrees in electrical engineering, from Purdue University, West Lafayette, IN, in 1977 and 1980, respectively.

He joined the faculty of Electrical and Computer Engineering at the University of Texas at Austin in 1982, where he is presently the Temple Foundation Professor (\#3) in Electrical Engineering. His research interests include applications of electromagnetic fields in tissues and in industrial processes, impedance measurements in tissues, and tissue thermal damage studies.

Dr. Pearce is a member of the International Microwave Power Institute, the American Society of Mechanical Engineers, Etta Kappa Nu, Tau Beta Pi, and Phi Kappa Phi. He is also the editor in chief of the Journal of Microwave Power and Electromagnetic Engineering. 